\title{
Erste Erfahrungen beim Aufbau der Zusammenarbeit der Technischen Fachhochschule Wildau mit der Universität „Sv. Kiril \& Metodij" Skopje
}

\author{
Gerhard Behrendt
}

\section{Der Weg zur Zusammenarbeit}

Der Zerfall des multiethnischen Jugoslawien führte zur Neugründung von kleineren Nachfolgestaaten und in der weiteren zeitlichen Folge zu einer Reihe militärischer Konflikte. Die Folgen des Kosovo-Krieges 1999 mit seinen Hunderttausenden von Flüchtlingen, die in den Nachbarstaaten Albanien, Mazedonien, Griechenland und Bulgarien Schutz suchten, die Belastungen dieser Staaten durch die Notwendigkeit, zusätzlich viele Menschen mit allem Erforderlichen zu versorgen, machten die Notwendigkeit der breiten Unterstützung durch die europäische Staatengemeinschaft deutlich. Die schnelle humanitäre Hilfe der EU-Mitgliedstaaten und vor allem der Bundesrepublik Deutschland zeigten aber auch, dass diese Soforthilfe in eine langfristige Unterstuitzung über die unmittelbare Beseitigung der Folgen von Krieg und Flüchtlingsströmen hinausgehen musste.

Anlässlich einer Beratung von Ministern der Republik Mazedonien und des Bundeslandes Brandenburg sowie interessierter Vertreter aus Wirtschaft und Wissenschaft im Brandenburgischen Landtag am 1. Juni 1999 wurde eine engere Zusammenarbeit in der Wirtschaft und beim akademischen Neuaufbau zwischen den Beteiligten vereinbart. In der Folge kam es zu einem ersten Kontakt zur Universität „Sv. Kiril i Metodij“ in Skopje, der zentralen Universität der Republik Mazedonien, und im September 1999 zu einem ersten Besuch der Universität durch Vertreter des Landes Brandenburg.

Die Gespräche mit dem Rektor, Prof. Dr. Ancevski, und den Vizerektoren, Prof. Dr. Cvetanovski und Prof. Dr. Dimeski, unterstrichen den dringenden Handlungsbedarf. In den folgenden Monaten wurden Versuche zur Organisation der Zusammenarbeit der Universität „Sv. Kiril i Metodij“ und der Technischen Fachhochschule Wildau im Rahmen des Sofortprogramms für die humanitäre Hilfe unternommen. Alle Aktivitäten in Mazedonien wurden durch das „Büro Kolbow“ wesentlich unterstiitzt [1]. Das Ergebnis der Beratungen in Skopje war die Vereinbarung der folgenden Schritte:

- Vorbereitung zum Abschluss eines Studentenaustauschabkommens zwischen der Universität „Sv. Kiril i Metodij“ und der Technischen Fachhochschule Wildau,

- Vorbereitung von Anträgen für gemeinsame FuE-Vorhaben von Forschergruppen beider Hochschulen über das Bundesministerium für Bildung und Forschung (BMBF),
- Anträge im Rahmen von EU-Programmen zur Anpassung der Lehre und Forschung der Universität an europäische Standards,

- Ausloten weiterer Fördermöglichkeiten des DAAD zur Unterstuitzung der Kooperation auf dem Gebiet der Ingenieurwissenschaften.

Daraufhin wurde zunächst beim DAAD im Sonderprogramm „Akademischer Neuaufbau Südosteuropa“ ein Förderantrag gestellt, der zwei Ziele verfolgte:

1. Schneller Austausch von Hochschullehrern bzw. der Rektoren zur Abstimmung der Wege der Zusammenarbeit und

2. Aufnahme einer Diplomandin an der Technischen Fachhochschule Wildau als Beginn ihrer Diplomarbeit, in der sie die Zusammensetzung der Sickerwässer der Deponie Drisla mit einer Multiparametersonde und Testsätzen untersucht.

Der Antrag wurde innerhalb von nur drei Tagen genehmigt. Innerhalb dieses Kurzzeitprojekts erfolgte der Besuch der von der mazedonischen Seite ausgewählten Diplomandin, Maja Georgieva. Sie wurde im Labor für Umweltverfahrenstechnik der Technischen Fachhochschule Wildau in das Arbeiten mit Testsätzen und Kolorimeter eingeführt. Bei der Umwelt- und Ingenieurtechnik GmbH Dresden wurde sie mit der Methodik der kontinuierlichen Messung von Parametern im Wasser vertraut gemacht und in das Arbeiten mit der Multiparametersonde eingewiesen. Mit dieser Sonde wurde ein kontinuierliches Messprogramm mit den Parametern pH-Wert, Temperatur, Leitfähigkeit, Salinität, Trübung, Redox-Potenzial, Ammonium, Nitrat vom Sikkerwasser der Deponie Drisla (bei Skopje) durchgeführt.

Durch Vermittlung des Büroleiters des „Büros Kolbow“ in Skopje, Oberst Quaden, wurde der Transport der Sonde und der Testsätze nach Skopje mit der Bundesluftwaffe organisiert. Innerhalb weniger Tage erfolgte der Transport von Frankfurt/Main, so dass die Arbeiten zum 1. März 2000 auf der Deponie beginnen konnten. Das „Büro Kolbow“ unterstuitzte die Arbeiten auch weiterhin durch die Bereitstellung eines Laptops zur Erfassung und Verarbeitung der auf der Deponie gewonnenen Daten. Die Daten wurden per e-mail an die Technische Fachhochschule Wildau und an die UIT Umweltund Ingenieurtechnik Dresden $\mathrm{GmbH}$ zur Kontrolle und Auswertung übermittelt. 
Im Rahmen eines Besuchs von Hochschullehrern der Technischen Fachhochschule Wildau in Skopje wurden

- ein Entwurf für ein Abkommen über den Studentenaustausch,

- zwei Entwürfe für gemeinsame FuE-Vorhaben,

- ein Entwurf für einen Antrag innerhalb des TEMPUSProgramms der EU

beraten. Die Anträge wurden zur Einreichung fertiggestellt, das Austauschabkommen unterschriftsreif formuliert. Beim Gegenbesuch der Vizerektoren der Universität „Sv. Kiril i Metodij“ in Wildau im Februar 2000 erfolgte die feierliche Unterzeichnung des Abkommens über den Austausch von Studenten zwischen beiden Einrichtungen durch den Rektor der Universität „Sv. Kiril i Metodij“, Prof. Dr. Ancevski, und den Präsidenten der Technischen Fachhochschule Wildau, Prof. Dr. Ungvari.

\section{Das Konzept der Zusammenarbeit}

Das übergeordnete Ziel des Konzepts der Zusammenarbeit der kooperierenden Hochschulen im Rahmen des Vorhabens „Akademischer Neuaufbau Suidosteuropas“ ist, die Kenntnisse über die zur Verbesserung der Umweltsituation verfuigbaren Verfahren, Techniken, Methoden, Herangehensweisen und Möglichkeiten in gezielten interdisziplinären Studien der jungen Generation der Völker des Balkanraumes zur nachhaltigen Entwicklung dieses Gebietes verfügbar und nutzbar zu machen. Dieses Ziel läßt sich erreichen durch eine praxisorientierte Ausbildung, in die Elemente der regionenbezogenen Forschung und Entwicklung ebenso wie eine Mitarbeit junger Wissenschaftler in FuE-Vorhaben in Deutschland integriert sind, so dass durch die unmittelbare Beschäftigung mit der Materie Verständnis, Wissen und Anwendungsmöglichkeiten in systematischer Arbeit vermittelt werden.

Dieses übergeordnete Ziel soll im Rahmen der bewilligten Mittel durch mehrere Teilziele erreicht werden. Diese Teilziele erstrecken sich gemäß der durch den DAAD bestätigten Konzeption auf folgende Bereiche:

- Vertiefung von Kenntnissen der deutschen Sprache,

- „Schnupperkurse“ von fortgeschrittenen Studenten in Deutschland,

- Austausch von Diplomanden,

- Anfertigung von Master-Arbeiten und Dissertationen im jeweiligen Partnerland,

- Durchfuihrung gemeinsamer Forschungsarbeiten,

- Aufbau gemeinsamer Technologietransferstellen und

- Austausch von Dozenten auf relevanten Lehr- und Arbeitsgebieten.

Die Inhalte der Teilziele wurden so formuliert, dass die Anforderungen aus dem Austauschabkommen der Technischen Fachhochschule Wildau und der Universität „Sv. Kirill i Metodij“ erfuillt und eine Förderung der Zusammenarbeit in FuE-Vorhaben möglich wird. Das Interesse junger mazedonischer Wissenschaftler an einer Arbeit an deutschen Hochschulen ist sehr groß, die Möglichkeiten zur Übertragung des Wissens und die Erzielung des „Schneeballeffekts“ zum Aufbau einer langfristig wirkenden strukturellen Entwicklung sind guinstig.
Wesentliche Momente werden folglich auf drei Ebenen der Partnerschaft gesehen:

1. Austausch von fortgeschrittenen Studenten der ingenieurwissenschaftlichen Fachrichtungen zwischen einem Monat und einem Semester mit der Möglichkeit, dass diese Zeit im jeweils anderen Land an der Partnerhochschule vollwertig anerkannt wird. Ein bedeutender Aspekt ist die Einrichtung von modularen Studiengängen an beiden Hochschulen einschließlich fremdsprachlicher Vorlesungen.

2. Austausch von Diplomanden, die ihre Diplomarbeit auf relevanten Forschungsgebieten an der Partnerhochschule durchführen. Ein zukünftiges Ziel ist die Verleihung eines Doppeldiploms durch beide Hochschuleinrichtungen.

3. Durchführung von Forschungsvorhaben und Integration von Doktoranden, die entweder an der Brandenburgischen Technischen Universität Cottbus oder an der Heimatuniversität promovieren können.

In die Zusammenarbeit der Hochschulen werden von deutscher Seite von Beginn an mittelständische Unternehmen integriert. Gemäß dem Auftrag der Fachhochschulen werden die FuE-Vorhaben praxisnah und in Verbindung mit mindestens einem Unternehmen durchgefuihrt. Dieses Modell wird auch in der Kooperation mit den Hochschuleinrichtungen Südosteuropas wirksam mit dem Ziel, es auch in diesen Ländern zu nutzen und die Praxisnähe der universitären Ausbildung zu erhöhen.

Die bisherige bilaterale Kooperation der Universität „Sv. Kirill i Metodij“ und der Technischen Fachhochschule Wildau wurde damit erweitert: Über das DAAD Sonderprogramm „Akademischer Neuaufbau Südosteuropa“ wird ein umfangreiches Projekt zum Technologietransfer gefördert, in dem auf deutscher Seite die Technische Fachhochschule Wildau die Projektkoordination ausübt. In dieses Projekt sind neben der Universität „Sv. Kirill i Metodij" die Brandenburgische Technische und die Universität für Chemische Technologie und Metallurgie, Sofia (Bulgarien), einbezogen.

In Zusammenarbeit der Technischen Fachhochschule Wildau und der Universität „Sv. Kirill i Metodij“ wurde im Rahmen des TEMPUS-Programms der EU ein Antrag zur Entwicklung gemeinsamer neuer modularer Studiengänge eingereicht, in den die Montanuniversität Leoben (Österreich), die University Loughborough (England) und die Chalmers University Göteborg (Schweden) sowie deutsche mittelständische Unternehmen integriert sind.

\section{Die bisherigen Ergebnisse im Rahmen des Programms "Akademischer Neuaufbau Südosteuropas"}

Die derzeitige Situation an den südosteuropäischen Hochschuleinrichtungen unterscheidet sich sehr von der deutschen. Das betrifft z. B. die Aufgeschlossenheit gegenüber den Problemen des Landes und den Willen, an einer Verbesserung aktiv mitzuwirken, den Grad der Vermittlung von modernen Problemlösungen sowie die Zusammen- 
hänge von erforderlichen Innovationen und wirtschaftlicher Prosperität. Die Aktivitäten im Rahmen des Austauschprogramms und des Sondervorhabens „Akademischer Neuaufbau Süidosteuropas" zielen auf eine langfristig wirksame Wissensvermittlung zur Verbesserung der Lebensqualität in den Ländern Süidosteuropas.

Ein Meilenstein auf dem Weg der langfristigen Kooperation ist die Weiterfuihrung der Arbeiten auf der Deponie Drisla nach Abschluss der Diplomarbeit von Frau Georgieva im Rahmen eines FuE-Vorhabens gemeinsam mit der AZBi GmbH Berlin, in dem die Probenahme und Analytik von polyaromatischen Kohlenwasserstoffen (PAK) in Böden untersucht sowie die Methoden dafuir weiterentwikkelt werden. Sie wird auf der Basis der Ergebnisse dieser FuE-Arbeiten ihren Master-Grad erlangen.

Die Ergebnisse und die Auswertung der Messungen von der Deponie Drisla werden die Grundlage für ein modernes Sanierungskonzept für die Deponie bilden, das gemeinsam mit dem Deponiebetreiber, mit einem regionalen Unternehmen sowie einem deutschen Graduierten erarbeitet wird.

Als nächster Schritt wurde ein Projekt im Rahmen des Stabilitätspakts „Akademischer Neuaufbau Südosteuropa" vom DAAD bewilligt, so dass zunächst für das Jahr 2000 und die folgenden bis 2004 beträchtliche Mittel für die Ausfüllung der entwickelten Konzepte bereitstehen. Damit ist ein großer Schritt in die Richtung einer effektiven Kooperation mit den südosteuropäischen Partnern getan.

Innerhalb dieses DAAD-Projekts wurden folgende Aktivitäten abgewickelt:

1. Eine Gruppe von 15 Studenten der Universität „Sv. Kirill i Metodij“ und von fünf Studenten der Universität für Chemische Technologie und Metallurgie Sofia (Bulgarien) führten mit einheimischen und deutschen Dozenten einen sechswöchigen Sprachkurs in Skopje durch. Dazu wurde an der Universität „Sv. Kirill i Metodij" ein Sprachlabor nach den Standards der und mit umfangreicher Hilfe durch die Technische Fachhochschule Wildau aufgebaut.

2. Studenten dieser Gruppe kommen nach Wildau oder Cottbus und führen hier die praktischen Arbeiten für ihre Diplomarbeiten durch. Sie nehmen gleichzeitig an der Sprachausbildung Deutsch teil.

3. Zwei Absolventinnen und drei Diplomandinnen der Universität „Sv. Kirill i Metodij“ führen in Wildau und der Außenstelle der BTU Cottbus ab 1. 10. 2000 die Arbeiten für ihre Master-bzw. Diplomarbeiten durch.

4. Zwei Diplomandinnen der Universität für Chemische Technologie und Metallurgie Sofia (HTMU) bearbeiten ab 01.10.2000 ihre Diplomarbeiten in Wildau. Die Themen für die Diplomarbeiten wurden aus den gegenwärtig laufenden FuE-Vorhaben herausgelöst und sind eng mit der Anwendung in mittelständischen Unternehmen des Landes Brandenburg verbunden.

5. Im Oktober 2000 beginnt der Austausch von Hochschullehrern zwischen den Einrichtungen. Dazu werden zunächst drei Professoren aus Skopje nach
Wildau und Cottbus reisen und Gastvorlesungen halten; im Gegenzug sollen im November Professoren der deutschen Einrichtungen in Skopje bzw. Sofia Gastvorlesungen halten.

Neben dem DAAD-Projekt werden in zwei FuE-Vorhaben (Förderung durch das Internationale Büro (IB) des BMBF) die folgenden Themen gemeinsam bearbeitet:

- Entwicklung von Kompositwerkstoffen auf der Basis Holz- bzw. Faser- und Kunststoffabfällen,

- Entwicklung von Kompositbaustoffen auf der Basis Holzabfälle und mineralischer Bindemittel.

Ein Vorhaben mit der Universität für Chemische Technologie und Metallurgie Sofia ist seit April 2001 zum Thema

- Entwicklung von Klebstoffen für die Schuhindustrie aus Recyclingpolyolen

durch das IB des BMBF bewilligt worden.

Bis zum Februar 2001 wurde das TEMPUS-Projekt zum Thema „Technologietransfer und modularer Studiengang" ausgearbeitet, während des Aufenthalts der Vizerektoren der Universität „Sv. Kirill i Metodij“ in Wildau der Text weitestgehend abgestimmt, so dass die redaktionelle Bearbeitung in Skopje innerhalb weniger Tage abgeschlossen und der Antrag im Februar 2001 eingereicht wurde.

Bei der Erarbeitung des TEMPUS-Antrages trat eine besondere Schwierigkeit auf, die in der relativen Selbständigkeit der Fakultäten der Universität „Ss. Kyrill und Methodij“ liegt und die durch das Rektorat nur sehr schwer zu Aktionen zu bewegen sind. Dieser hohe Grad an Selbständigkeit hat in einem gewissen Maße zu einer Verselbständigung gefuihrt, die innerhalb der Universität einer Autarkie gleichkommt. Die Kooperation mit mehreren Fakultäten erfordert demzufolge Verhandlungen mit jeder Fakultät und mit dem Rektorat.

Das Konzept des Projekts „Entwicklung einer praxisorientierten Ausbildung auf dem Gebiet der Umwelttechnik" ist auf folgende Ergebnisse angelegt:

- Ein vertieftes Verständnis der Teilnehmer aus den beteiligten Staaten und Einrichtungen für die Probleme des jeweiligen Gegenuibers, insbesondere eine weitergehende Kommunikation der jungen Generation und deren Integration in die Prozesse des Neuaufbaus der Gesellschaft in den südosteuropäischen Ländern.

- Ein Beitrag zur Strukturentwicklung in der Region, durch die eine weitergehende Stabilisierung der Gesellschaft und ein wirtschaftlicher Aufschwung initiiert werden. Dadurch werden Grundlagen für eine nachhaltige Entwicklung durch Vernetzung der Hochschuleinrichtungen mehrerer südosteuropäischer Staaten in der gesamten Region geschaffen.

- Die Kooperation der Hochschulen in Südosteuropa mit mittelständischen Unternehmen und Landwirtschaftsbetrieben wird unterstuitzt und der Aufbau internationaler Netzwerke gefördert.

- Durch die Kooperation mit Unternehmen, die bereits Kooperationspartner der deutschen Hochschulen 
sind, werden neue internationale Beziehungen aufgebaut und auf diese Weise neue Ansatzpunkte für eine Expansion von Unternehmen geschaffen.

\section{Bisherige wissenschaftliche Ergebnisse}

Innerhalb des vom DAAD geförderten Vorhabens wurde eine Diplomarbeit abgeschlossen, fünf Diplomarbeiten wurden zum 30. April bzw. 30. Juni 2001 bearbeitet. Hinzu kommen zwei Master-Arbeiten, für die im Januar die Arbeit begonnen wurde.

Die Diplomarbeiten sind eng mit umweltrelevanten Entwicklungen, die im Institut für Kreislaufwirtschaft im Rahmen von FuE-Vorhaben mit mittelständischen Unternehmen der Region bearbeitet werden, verknüpft. Sie sind auf die Entwicklung neuer Materialien unter anderem aus Produktionsreststoffen ausgerichtet. Im Rahmen der Master-Arbeiten werden Untersuchungen von anthropogenen Einflüssen auf die Umwelt durch Altablagerungen untersucht und dazu eine Methodologie entwickelt.

\section{1 „Synthese von Polyolen für Polyurethanbeschich- tungssysteme durch kombinierte Aminolyse/Glyko- lyse von Weichschaumstoffen"}

Inhalt der Arbeit ist, aufbauend auf dem an der Technischen Fachhochschule Wildau erarbeiteten Wissensstand, eine Synthese für Polyole zu entwickeln, die die Herstellung von Beschichtungssystemen auf der Basis Polyurethan erlaubt. Dazu werden unterschiedliche Verhältnisse an Glykolen, sekundären aliphatischen Aminen und PUR-Weichschaumstoffen verwendet. Die Reaktion verläuft bei optimaler Rezepturgestaltung innerhalb von 30 Minuten, so dass die Spaltung der Polyharnstoffe aus der Isocyanat-Wasser-Reaktion durch die Hydroxylgruppen der Glykole reduziert und damit die Bildung primärer aromatischer Amine unterdrückt wird. Die Glykole werden so ausgewählt, dass sie ein gutes Lösungsvermögen für die Polyharnstoffe bei einer angepassten Reaktivität gegenüber Isocyanatgruppen aufweisen. Die Polyole werden durch Viskosität, Hydroxylzahl, Aminzahl und Amingehalt (bestimmt durch GC-MS) charakterisiert.

Die bisher erhaltenen Polyole liegen im Bereich der Hydroxylzahlen zwischen 360 und 200 sowie in den Viskositäten zwischen 4.000 und $18.000 \mathrm{mPas}\left(25^{\circ} \mathrm{C}\right)$. Die Aminzahlen konnten durch die Variation in der Rezeptur und in den Reaktionsbedingungen unter 20 gesenkt werden, d. h. das sekundäre aliphatische Amin reagiert nahezu quantitativ mit den Urethangruppen zu trisubstituierten Harnstoffen. Aus den Polyolen wurden mit Polyisocyanaten Beschichtungen auf dem Labcoater mit einer Folienstärke von $4 \mathrm{~mm}$ hergestellt. Die Shore-A-Härte der Folien liegt zwischen 80 und 95. Weitere mechanische Eigenschaften werden geprüft (Zugfestigkeit, Bruchdehnung, Kraft-Dehnungs-Diagramme) und durch DMA die Morphologie der Folien charakterisiert [2].
4.2 „Untersuchungen zur Dispergierung von Melaminderivaten in Polyesterpolyolen unterschiedlicher Struktur“

Inhalt der Arbeit ist, aufbauend auf dem an der Technischen Fachhochschule Wildau erarbeiteten Wissensstand, eine Synthese für Polyole auf der Basis von PET-Produktionsabfällen und Melaminderivaten wie Melaminharzpulvern aus der Leiterplattenproduktion bzw. Melamincyanurat zu entwickeln. Die Polyesterdispersionspolyole werden zur Herstellung von flammgeschützten Polyurethan-Hartschaumstoffen verwendet. Der erste Schritt besteht in der Synthese von Polyesterdiolen mit niedriger Viskosität durch gemeinsame Kondensation der als Abfälle zur Verfuigung stehenden Oligokondensate mit verschiedenen Glykolen und aliphatischen Dicarbonsäuren [3]. Zur Synthese werden unterschiedliche Verhältnisse an Oligoesterkondensaten, unterschiedlichen Glykolen, aliphatischen Dicarbonsäuren und gegebenenfalls PET-Flaschenabfällen verwendet. Die Polyole werden durch Viskősität, Hydroxylzahl und Säurezahl charakterisiert. Die Viskositäten der bisher hergestellten Grundpolyole liegen im Bereich von 8.000 bis $1.400 \mathrm{mPas}\left(25^{\circ} \mathrm{C}\right)$, die Hydroxylzahlen zwischen 180 und $500 \mathrm{mg} \mathrm{KOH} / \mathrm{g}$ und damit in einem Bereich, der eine Dispergierung von Feststoffen zulässt. In den Polyolen werden mit verschiedenen Technologien Melaminharzpulver bzw. Melamincyanurat nach geeigneter Aufbereitung durch Vermahlen und Bestimmung der Teilchengrößenverteilung dispergiert. Aus den Dispersionspolyolen werden anschließend mit Polyisocyanaten Hartschaumstoffe mit der Laborschäummaschine hergestellt. Die Hartschaumstoffe werden mechanisch geprüft (Druckfestigkeit, Dimensionsstabilität bei $120^{\circ} \mathrm{C}$, Schlagzähigkeit); die Flammfestigkeit nach ASTM 1694 sowie mittels Sauerstoffindex (LOI-Wert) bestimmt.

\section{3 „Untersuchung der Wirkung von thermoplasti- schen Polyurethanen als Verträglichkeitsvermittler im System PET-PP“}

Durch die Synthese von speziellen thermoplastischen Polyurethanen auf der Basis von TerephthalsäuresterOligomeren mit Polyisobutadienpolyolen bzw. ähnlicher, hydroxylfunktionalisierter Kohlenwasserstoffabkömmlinge [4] werden neuartige Verbindungen mit polaren und unpolaren Strukturelementen hergestellt. Für diese Polymeren sind mittlere Molmassen oberhalb $4.000 \mathrm{~g} / \mathrm{Mol}$ Voraussetzung zur Erzielung eines hohen Eigenschaftsniveaus der Basispolymeren. Die bisher erzielten Molmassen liegen bei ca. 5.000, sind aber bereits zur Einarbeitung in Polymerlegierungen geeignet. Diese thermoplastischen Polyurethane sollen in bestimmten Verhältnissen PET-PP-Mischungen zugesetzt und die DreiKomponenten-Mischungen auf der Brabender LabStation zu Drähten extrudiert werden. An den Drähten der Mischungen werden thermomechanische und elektronenmikroskopische Untersuchungen zur Bestimmung der Homogenität und der Morphologie durchgeführt, weiterhin werden die thermomechanischen Werte durch DMA bestimmt und aus den Relaxationsspektren die Anzahl und der Anteil der Phasen berechnet. 


\section{4 „Untersuchung der Wirkung von thermoplasti- schen Polyurethanen als Verträglichkeitsvermittler im System PET-PVC““}

Inhalt der Arbeit ist, die Synthese von speziellen thermoplastischen Polyurethanen auf der Basis von Terephthalsäurester-Oligomeren mit halogenhaltigen Diolen. Dazu werden zunächst Homopolymerisate kurzkettiger Diole durch kationische Polymerisation mit einer gezielt eingestellten Molmasse und einem bestimmten Chlorgehalt hergestellt. Durch die Kettenlänge der chlorhaltigen Polymerisate wird eine Durchdringung der molekularen Oberflächenschichten der PVC-Phase erreicht, während die Polyesterurethankomponente mit der PETPhase verträglich ist und sich bei ausreichender Kettenlänge mit dieser mischt [4]. Auf diese Weise wird eine dritte Phase in den Polymerlegierungen erzeugt. Die thermoplastischen Polyurethane werden in bestimmten Verhältnissen PET-PVC-Mischungen zugesetzt und diese Drei-Komponenten-Mischungen auf der Brabender LabStation zu Drähten extrudiert. An den Drähten der Mischungen werden thermomechanische und elektronenmikroskopische Untersuchungen zur Bestimmung der Homogenität und der Morphologie durchgeführt. Aus den thermomechanischen Werten werden die Anzahl und der Anteil der Phasen berechnet.

\section{5 „Entwicklung von Formstoffen aus biologisch abbaubaren Kunststoffen und nachwachsenden Rohstoffen"}

Durch Nutzung geeigneter Synthesewege zur radikalischen Pfropfung olefinischer Monomerer mit RedoxSystemen werden gepfropfte modifizierte Stärke und Cellulose hergestellt, deren Eigenschaften derart ausgewählt werden, dass sich die Produkte durch Extrusion oder Spritzguss verarbeiten lassen. Die Pfropf-Monomere werden so ausgewählt, dass biologisch abbaubare Polymerformteile zunächst auf Basis von Stärkeprodukten hergestellt werden. In der zweiten Phase werden die modifizierten Stärkeprodukte zusammen mit Fasern oder Blähkörpern aus Hanf, Flachs oder Roggen durch Extrusion und Spritzguss zu biologisch schnell abbaubaren, kurzzeitig nutzbaren Formkörpern mit kurzen Kompostierzyklen verarbeitet. Aus den Formkörpern werden außerdem Prüfmaterialien gewonnen, an denen physikalische und thermomechanische Werte bestimmt sowie die Kompostierbarkeit untersucht werden.

\section{6 „Einfluß von physikalischen und chemischen Ma- terialeigenschaften auf das Migrations-, Adsorptions- und Abbauverhalten der PAK in ausgewählten Böden“}

Der Inhalt der Arbeit ist auf die Vertiefung der bisher erworbenen Kenntnisse zur Untersuchung von Böden und Deponien gerichtet. Die Arbeiten wurden im Jahre 2000 mit Messungen auf der Deponie Drisla in Mazedonien begonnen. Im Rahmen eines größeren FuE-Vorhabens werden die Untersuchungen auf die spezielle PAKProblematik als eines der häufigeren Fälle im Boden sowie im Deponiesickerwasser durch Feldversuche und Bestimmungen der PAK in Bodenprofilen gerichtet. Dabei wird der Beschreibung von Matrixeinflüssen auf PAKMessparameter große Bedeutung zugemessen und darüber hinaus weitere Bodenparameter wie der pH-Wert, die elektrische Leitfähigkeit, das Redox-Verhalten, der Glühverlust, die Kornverteilung, der Tonanteil, der Gesamtkohlenstoff und der CSB am Sickerwasser bestimmt. An Lysimetern werden mit ausgewählten Böden unter Einsatz von Multiparametersonden Elutionsuntersuchungen zur Bestimmung des Elutionsverhaltens von PAK in verschiedenen Böden mit unterschiedlichen Elutionsmitteln durchgeführt und in Modellversuchen die Wiederfindungsrate bestimmt.

\section{Planungen für die Zukunft}

Das vom DAAD geförderte Projekt wird unter Einbeziehung vieler Mitarbeiter der Technischen Fachhochschule Wildau weiter im Sinne des Leitgedankens des Programms umgesetzt. Dazu stellt der DAAD jährlich beträchtliche Mittel zur Verfuigung, die zukünftig auch von deutschen Studenten, Graduierten und Professoren genutzt werden sollen. Die bisherigen Erfahrungen in der Zusammenarbeit mit der Universität „Sv. Kirill i Metodij“ werden dabei beachtet und der zeitliche Vorlauf für gemeinsame Aktionen gegenüber der Anlaufphase des auf fünf Jahre konzipierten Projekts wesentlich vergrößert, um in beiden Ländern eine sinnvolle Auswahl der Kandidaten zu gewährleisten.

Darüber hinaus wurden zwei weitergehende TEMPUSAnträge in das Programm aufgenommen, von denen einer 2001 und der andere 2002 eingereicht werden. Diese Anträge sollen zum Inhalt haben:

1. Den Aufbau einer Technologie-Transferstelle kombiniert mit dem Aufbau eines Intranet an der Universität „Sv. Kirill i Metodij“, durch das alle relevanten Fakultäten vernetzt werden und auf diesem Wege eine interdisziplinäre Zusammenarbeit unterstiutzt wird, gleichzeitig eine Intensivierung der Curricula des Instituts für Interdisziplinäre Studien erfolgt und mehr Studenten in diese Ausbildung integriert werden.

2. Die Entwicklung eines gemeinsamen, internationalen Studienganges, der mit einem Doppeldiplom abschließen wird, und Erprobung des Bachelor/MasterSystems in diesem Studiengang.

Die bisherige Arbeit im Rahmen des DAAD-Programms soll mit zusätzlichen individuellen FuE-Vorhaben unterstützt werden. Die Möglichkeiten der staatlichen Kooperation werden über das IB des BMBF verstärkt genutzt auf den von beiden Regierungen im Abkommen festgelegten prioritären Aufgabengebieten. Für die Jahre 2000 bis 2003 wurden zwei gemeinsame FuEVorhaben bewilligt:

1. Entwicklung von Verbundwerkstoffen aus Holzspänen und Polyurethansystemen. Dieses Vorhaben ist auf neue Werkstoffe als Alternative zu herkömmlichen Spanplatten (MDF usw.) gerichtet, um diese aus in Mazedonien vorhandenen Rohstoffen ohne um- 
weltschädliche Lösungs- oder Bindemittel mit einer einfachen Technologie gemeinsam mit deutschen Unternehmen herzustellen.

2. Entwicklung von Basiselementen für Maschinen aus anorganisch hochgefüllten Polymeren. In diesem Vorhaben sollen Basiselemente für Maschinen, die derzeit aus Druckguss hergestellt werden, durch Kompositwerkstoffe aus Quarzsand und speziell dafür entwickelten Polymeren - Polyestern, Polyepoxiden, Polyurethanen - entwickelt und auf ihre Beständigkeit unter dem Einfluss von Schwingungen sowie Ölen untersucht werden.

Die Erfahrungen im zurückliegenden Jahr haben die große Bereitschaft der Beteiligten zu einer Kooperation gezeigt. Die bisherigen Ergebnisse wurden durch ein hohes Maß von Engagement von allen Beteiligten möglich. So manche uniberwindlich scheinenden Schwierigkeiten wurden durch die Kooperation mit dem Büro Kolbow und der Bundesluftwaffe schnell beseitigt, manchmal Unmögliches möglich gemacht, insbesondere wenn es um simple Transportaufgaben im Lande und in das Land ging. Dieser Schwung engagierter Menschen sollte als Beispiel dienen und andere ansprechen, sich stärker und intensiver fuir eine Stabilisierung der Region einzubringen. Von den beteiligten Mitarbeitern der Technischen Fachhochschule Wildau wurde der Weg gewählt, durch Beispiele in der universitären Ausbildung junge Menschen zusammenzubringen und die Fundamente für neue Ausbildungsmodelle sowie integrative Maßnahmen zu legen. Das ist eine von vielen Möglichkeiten zum Wiederaufbau der Region, aber auch durch die Nutzung der Multiplikatoren „Absolventen“ eine vielversprechende, die weitere Unterstiitzung und Mitarbeit finden sollte.

\section{Teilnehmer am Ausíauschprogramm}

\subsection{Teilnehmer aus der Universität „Sv. Kiril i Metodij“ Skopje}

Prof. Dr. Dimeski, Vizerektor

Prof. Dr. Cvetanovski, Vizerektor

Prof. Dr. Cepujnoska, Vizerektorin

Prof. Dr. Glamocanin, Dekan der Fakultät für

Elektrotechnik

Prof. Dr. Dudeski, Fakultät für Maschinenbau

Prof. Dr. Jovanovski, Leiter des Instituts für Verfahrenstechnik

Prof. Dr. Popovski, Fakultät für Elektrotechnik

Prof. Dr. Spaseska, Fakultät für Technologie und Metallurgie

Dr. B. Iliev, Fakultät für Forstwissenschaften

Dipl.-Ing. G. Vrtanovski, Fakultät für Maschinenbau

Dipl.-Ing. M. Georgieva, Institut für interdisziplinäre

Studien der Umweltwissenschaft

Dipl.-Ing. I. Šišovska, Institut für interdisziplinäre

Studien der Umweltwissenschaft

cand. ing. S. Petrovska, Fakultät fuir Maschinenbau cand. ing. G. Nikolovska, Fakultät für Technologie und Metallurgie

cand. ing. K. Nikolovska, Fakultät für Technologie und Metallurgie

\subsection{Teilnehmer der Universität für Chemische Technologie und Metallurgie, Sofia}

Prof. Dr. Evtimova, Institut für Schuh- und Lederindustrie Dr. Pischev, Dekan der Fakultät für Chemische Verfahrenstechnik

cand.-ing. S. Ivanyi, Fakultät für Chemische

Verfahrenstechnik

cand.-ing. J. Lozeva, Fakultät für Chemische

Verfahrenstechnik

\subsection{Teilnehmer der Technischen Fachhochschule Wildau}

Prof. Dr. Ungvári, Präsident der TFH Wildau

Prof. Dr. G. Behrendt, FB Ingenieurwesen/Wirtschaftsingenieurwesen

Prof. Dr. Ebner, FB Ingenieurwesen/Wirtschaftsingenieurwesen

Dr. H.-D. Hunger, Institut für Kreislaufwirtschaft

Dr. R.Langenstraßen, Institut für Kreislaufwirtschaft

Dr. A. Schubert, Akademisches Auslandsamt

Dr. habil. W. Ecknig

Dipl.-Geol. R. Obst

\subsection{Brandenburgische Technische Universität, Cottbus}

Prof. Dr. D. Möller, Lehrstuhl für Luftchemie und Luftreinhaltung

\section{Ausblick}

Die Fortsetzung des Vorhabens in den Jahren bis 2005 mit einer jährlichen Fördersumme von über 200.000 DM erfordert die aktive Teilnahme auch der deutschen Seite. Wenn die Situation in Mazedonien gegenwärtig auch schwierig ist, so besteht für Hochschullehrer, Mitarbeiter und Studenten die Möglichkeit kurz- und längerfristiger Aufenthalte in Skopje oder Sofia. Es ist eine Bedingung zur erfolgreichen Durchführung des Vorhabens, daß diese Möglichkeiten genutzt werden. Insbesondere sollten auch Studenten der beteiligten Hochschulen die Chance nutzen, an der Universität „Sv. Kiril i Metodij“ oder der Universität für Chemische Technologie und Metallurgie ihre Diplomarbeiten durchzuführen oder an Master-Studiengängen teilzunehmen.

Jährlich werden aus den beteiligten Ländern etwa 15 Hochschullehrer in Deutschland sein. Hinzu kommen jährlich bis zu 10 Diplomanden/innen bzw. Graduierte für eine höhere Ausbildung. Die Interessen der Hochschullehrer liegen vor allem auf den Gebieten moderner Technologien, neuer Materialien, Informationstechnik, Hochschulmanagement und alternativer Energiesysteme. Über die Universitäten und ihre Hochschullehrer können darüber hinaus Kontakte zu mittelständischen Unternehmen geknuipft werden, so dass daraus Vernetzungen mit deutschen Unternehmen entstehen. Der Aufbau von Kooperationen auf dem Gebiet in- 
novativer Technologien, die Nutzung von FuE-Ergebnissen deutscher Forschergruppen aus den beteiligten Einrichtungen in diesen und der Marktzugang in Südosteuropa über die beiden Länder sollte ein weiterer wichtiger Aspekt der internationalen Zusammenarbeit der öffentlichen Einrichtungen des Landes Brandenburg sein.

\section{Literałur}

[1] G. Behrendt in: Wir sind alle gefordert, Makedonien - ein Testfall, Hrsg. W. Kolbow und H. Quaden, Nomos-Verlag, Baden-Baden, 2001

[2] K.-H. Schmidt, H. Koch, M. Pohl, H. Huth, S. Ivanyi, O. Stürmer, G. Behrendt, Entwicklung von Polyurethan-Beschichtungssystemen auf der Basis von Recyclat-Polyolen aus Polyurethan-Weichschaumstoffen, Wiss. Beitr. Techn. Fachhochschule Wildau 2001

[3] G. Behrendt, M. Pohl, Polyesterpolyole mit niedriger Viskosität, DE-Anm. 19915128.8 vom 25. 03. 1999

[4] G. Behrendt, Verträgliche Polymerlegierungen und Verfahren zu ihrer Herstellung, DE-Anm. 10037434.4 vom 24.07.00

\section{Aułor}

\section{Prof. Dr. Gerhard Behrendt}

Technische Fachhochschule Wildau

Fachbereich Ingenieurwesen/Wirtschaftsingenieurwesen Tel. (0 33 75) 508-591

E-Mail: behrendt@vt.tfh-wildau.de 